\section{Les mécanismes moléculaires de la méiose chez les plantes}

Christine Horlow, Marie-Pascale Doutriaux
> La méiose est une étape-clé de la reproduction sexuée chez les organismes diploïdes. Au-delà de sa description cytologique, les mécanismes moléculaires qui entrent en jeu dans cette division spécialisée commencent à être décryptés chez les plantes grâce à la plante-modèle Arabidopsis thaliana. Si certains mutants méiotiques d'Arabidopsis confirment le rôle de fonctions centrales déjà décrites, par exemple, chez la levure ou chez la souris, d'autres ont permis d'identifier des gènes dont la fonction est inconnue à ce jour. De nombreuses plantes existent aussi à l'état de polyploïdes, ce qui pose un problème particulier du point de vue de la méiose. < $(\rightarrow) \mathrm{m} / \mathrm{s}$ 2001, n० 8-9, p. 829
Loin de la discrétion observée chez les animaux, la différenciation des organes reproducteurs chez les plantes atteint, avec la fleur des angiospermes, une variété incroyable de formes, d'odeurs et de couleurs. Les stratégies de séduction ainsi élaborées visent les insectes pollinisateurs qui sont les vecteurs de la reproduction et participent à la dissémination des caractères génétiques des plantes. Les fleurs n'ont pas manqué d'attirer aussi l'attention des humains et sont devenues un sujet d'étude fort prisé des cytogénéticiens du fait, notamment, de l'abondance et de l'accessibilité du matériel produit dans les organes reproducteurs mâles (anthères). C'est dans le tout jeune bouton de fleur que se déroule la méiose, étape essentielle de la reproduction sexuée des organismes diploïdes. Au cours de cette division particulière, une cellule diploïde réduit son matériel chromosomique de moitié pour produire quatre cellules haploïdes. La ségrégation correcte des chromosomes lors de la méiose dépend de leur association en paires d'homologues, ce qui est l'occasion de remaniements des associations de caractères génétiques dont ils sont porteurs. La méiose ouvre donc une voie à la diversification des individus au sein d'une espèce. Depuis longtemps, les humains ont exploité cette variabilité, améliorant ainsi, par sélections et croisements

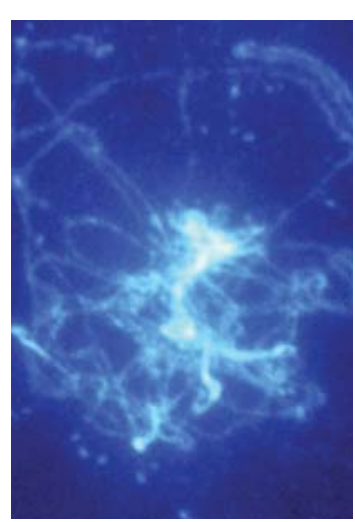

incessants, les qualités de plantes qui constituent une source alimentaire essentielle.

Malgré les nombreuses informations accumu-

lées sur la reproduction sexuée des espèces végétales cultivées, la taille de leur génome et leur niveau de ploïdie ont représenté un obstacle à l'adaptation des outils de la biologie moléculaire et de la génomique fonctionnelle. Les efforts de la communauté scientifique du végétal se sont alors focalisés sur l'étude d'une plante modèle, Arabidopsis thaliana $(\rightarrow)$. Cette crucifère a été choisie pour ses qualités de croissance rapide, de faible encombrement spatial, son caractère autogame et sa prolificité (Figure 1). Mais surtout, la taille réduite de son génome (125 Mb; 25000 gènes) a permis son séquençage complet [1]. L'analyse de ce génome montre que la plupart des fonctions impliquées dans la méiose chez la levure ou chez la souris présentent des homologues chez Arabidopsis. La convergence de phénotypes des mutants méiotiques dans les règnes du vivant suggère un niveau élevé de conservation de cette division particulière qui est à l'origine de la reproduction sexuée. 


\section{Physiologie de la reproduction chez les plantes}

La mise à fleur intervient après que la plante a atteint un stade de maturité donné, et répond à des conditions environnementales précises (photopériode, intensité lumineuse, température...). Selon les espèces, la fleur contient l'un ou l'autre ou les deux organes mâle (anthères) et femelle (les ovules logés dans le pistil). Dans ces organes, des cellules spécialisées, les méiocytes subissent la méiose qui donne lieu à la production de quatre microspores (mâle) ou mégaspores (femelle) haploïdes. À l'issue de la méiose, dans les anthères, chaque microspore subit une ou deux mitoses (selon les espèces) lors de sa différenciation en un grain de pollen. Dans les ovules, une seule des quatre mégaspores se développe, après trois mitoses successives, en un sac embryonnaire constitué de 8 noyaux et de 7 cellules parmi lesquelles l'oosphère (Figure 2). II est à noter que la différenciation des méiocytes se produit chez les plantes à partir de cellules qui ont éventuellement subi une très longue période végétative. II n'y a pas, comme chez les mammifères, de lignée reproductive précocement déterminée, protégée de cycles mitotiques récurrents et des aléas de l'environnement.

\section{La méiose chez Arabidopsis}

Les différents stades de la méiose sont communs à la plupart des organismes diploïdes. Les chromosomes, qui ont subi une étape de réplication pré-méiotique, comportent deux chromatides-sœurs lorsque commence la méiose. L'organisation spécifique des chromosomes conditionne leur répartition correcte au cours des deux divisions successives de la méiose. En effet, selon un enchaînement très précis, les chromosomes homologues s'associent en bivalents puis ségrègent lors de la première division (méiose I, réductionnelle), tandis que les chromatides-sœurs se séparent seulement lors de la deuxième division (méiose II, équationnelle). L'appariement des chromosomes, la mise en place du complexe synaptonémal et le déclenchement de la recombinaison homologue ont lieu lors de la prophase
I et sont essentiels à la ségrégation correcte des chromosomes homologues. L'avancement dans cette phase peut être suivi cytologiquement en fonction de l'état des chromosomes ou des structures qui leur sont associées. Les chromosomes se condensent progressivement pendant les stades leptotène et zygotène. Au stade pachytène, le complexe synaptonémal est en place, tandis que les chromosomes poursuivent leur condensation. Au stade diplotène, le complexe synaptonémal se dépolymérise, libérant les chromosomes sauf au niveau des chiasmas (points de jonction ou crossing-over). En fin de prophase I, les chromosomes associés en bivalents présentent la structure en $X$ typique du stade diacynèse. Les bivalents se positionnent alors sur la plaque métaphasique avant leur ségrégation en deux lots chromosomiques équivalents (méiose I, réductionnelle). La méiose II (équationnelle) s'ensuit avec la perte de cohésion des chromatides sœurs et leur séparation qui aboutit à la partition du lot chromosomique initial en quatre génomes haploïdes. La succession de ces différentes étapes a pu être décrite chez Arabidopsis malgré la petite taille de ses cinq paires de chromosomes, ouvrant ainsi la voie à la caractérisation de fonctions impliquées dans les processus méiotiques [2] (Figure 3).

De nombreux mutants de plantes ont été isolés sur le critère de leur stérilité plus ou moins drastique. Parmi ceuxci, les mutants méiotiques associent en général le phénotype de stérilité (souvent mâle et femelle) à des déviations du nombre et de la taille des microspores au stade tétrade, et à des défauts de développement du sac embryonnaire (Figure 1). Chez Arabidopsis, il est devenu
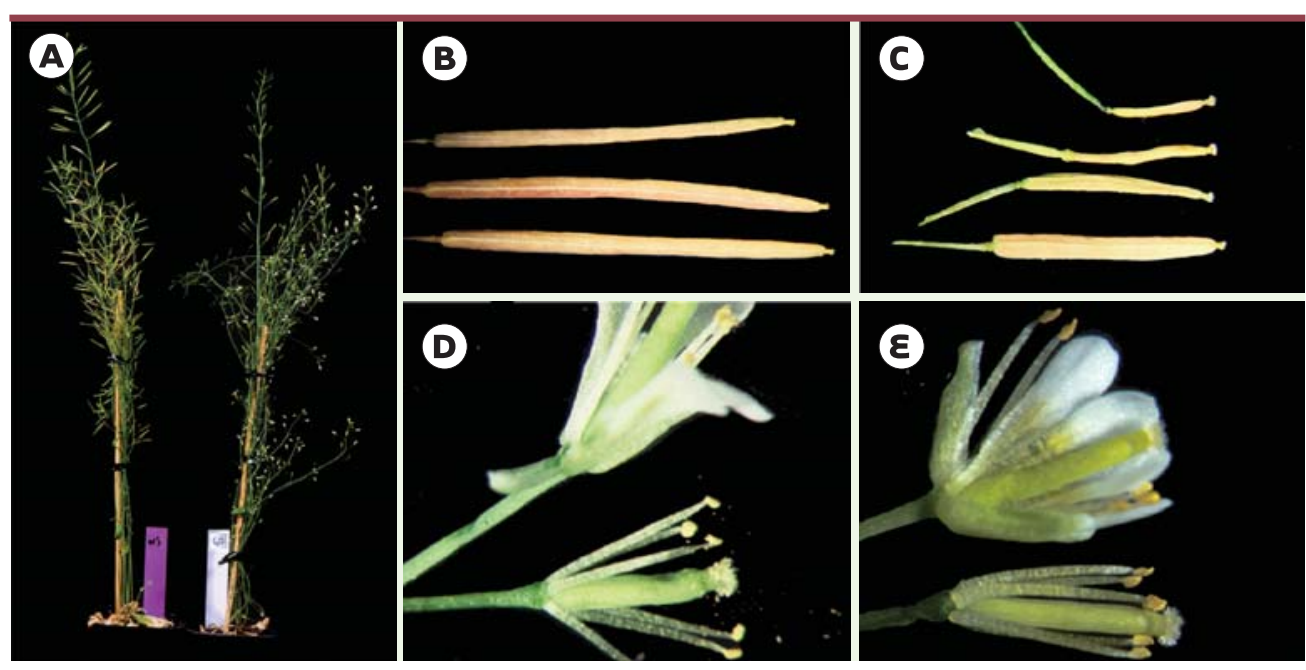

Figure 1. Arabidopsis thaliana. A. Plantes entières; à gauche une plante sauvage et à droite une plante mutante méiotique $d m c l$ dont les fruits partiellement stériles sont plus courts que ceux de la plante sauvage. $\boldsymbol{B}$. Siliques (ou fruits) de plantes sauvages. $C$. Les siliques de mutant $d m c l$ sont plus courtes que celles de la plante sauvage. D. Fleur d'Arabidopsis sauvage. $\varepsilon$. Fleur d'Arabidopsis dmcl : les anthères contiennent très peu de pollen par rapport à celles de la plante sauvage. 
possible de caractériser moléculairement les fonctions défectueuses de ces mutants du fait, notamment, du recours à la mutagenèse insertionnelle et aux clonages positionnels. Ainsi, à côté de mutants de fonctions précédemment décrites chez d'autres espèces (spoll, asyl, syn1), ont pu être décrits aussi des mutants de fonctions sans équivalent connu à ce jour (swil, ms5). De plus, les collections de mutants ont permis des approches de génétique inverse qui, partant de la séquence connue d'un gène, mènent à l'isolement du mutant correspondant (dmcl, atm).

\section{Le complexe synaptonémal}

Le complexe synaptonémal est une structure protéique particulière qui apparaît (stade zygotène) puis disparaît (diplotène) au cours de la prophase I de méiose. Au stade pachytène, le complexe synaptonémal semble établir une connexion entre les chromosomes homologues sur toute leur longueur. La mise en place d'un complexe synaptonémal cohérent est nécessaire à la formation des bivalents chez Arabidopsis. En son absence, le stade pachytène ne peut être défini chez le mutant asyl, qui ne présente pas non plus de bivalents en métaphase I. Le produit du gène AtASYl (At: Arabidopsis thaliana) est homologue de la protéine Hopl de levure, elle-même nécessaire à la formation du complexe synaptonémal chez la levure [3]. Ce travail confirme la conservation de fonctions entre la levure et Arabidopsis.

\section{La recombinaison homologue}

La recombinaison homologue méiotique intervient lors de la prophase l et concrétise les interactions entre chromosomes homologues en stabilisant les bivalents. Une étude des fréquences de recombinaison homologue montre une distribution non aléatoire des crossing-over méiotiques chez Arabidopsis, qui assure la présence d'au moins un crossing-over par chromosome [4]. Le lien entre réparation des lésions de l'ADN, recombinaison homologue et méiose est avéré chez Arabidopsis puisque plusieurs mutants (non caractérisés) hypersensibles aux rayons $X$ sont aussi affectés dans la recombinaison homologue méiotique et partiellement stériles [5].

Chez la levure, la protéine Spoll induit la formation des cassures double-brin nécessaires à la mise en route de la recombinaison homologue méiotique tandis que la protéine Dmcl est spécifiquement impliquée dans l'orientation de la recombinaison réparatrice vers le chromosome homologue (et non la chromatide-sœur). Les mutations des gènes AtSPOIl et AtDMCl entraînent une stérilité partielle chez Arabidopsis, confirmant le rôle de la recombinaison homologue pour la formation des bivalents chez cette plante. Les plantes mutantes spoll présentent une diminution drastique de la recombinaison homologue méiotique. De ce fait, il n'y a pas formation de bivalent et les dix chromosomes, à l'état d'univalents, ségrègent aléatoirement [6]. Ce phénotype est similaire à celui des plantes mutantes $\mathrm{dmcl}$ (Figure 4) [7]. Ces résultats suggèrent fortement que le déclenchement de la recombinaison homologue méiotique repose sur la création de cassures double-brin parla protéine Spoll chez les plantes, et que leur réparation par Dmcl conditionne la formation des bivalents. Les plantes mutantes spoll ne présentent pas de stade pachytène discernable ( $F i$ gure 4), ce qui indique que l'établissement du complexe synaptonémal dépend du déclenchement de la recombinaison homologue chez Arabidopsis comme chez la souris et chez la levure.

\section{La cohésion des chromatides sœurs}

anthères, un méiocyte mâle (cellule mère du pollen) donne naissance, à l'issue de la méiose, à 4 microspores qui se différencient en 4 grains de pollen. Chaque grain de pollen contient un gamète mâle ou cellule spermatique. Dans les ovules, une cellule mère des mégaspores donne naissance, à l'issue de la méiose, à 4 mégaspores dont une seule se différencie en 1 sac embryonnaire. Le sac embryonnaire contient le gamète femelle ou oosphère.
La méiose étant précédée par une étape de réplica- 
tion, les chromosomes entrent en méiose constitués de deux chromatides dont la cohésion doit être maintenue tout au long de la méiose I. En effet, c'est seulement lors de la deuxième division (méiose II) que les chromatidessœurs se séparent.

Le phénotype des mutants swil d'Arabidopsis est interprété comme résultant d'un défaut de cohésion des chromatides-sœurs [8]. La mutation swil-2 entraîne des phénotypes divergents selon que les méiocytes sont mâles ou femelles: les méiocytes femelles swil-2 effectuent une division de type mitotique en lieu et place de la méiose (séparation équilibrée des chromatides-sœurs en $2 \times 10$ univalents); dans les méiocytes mâles swil-2, les 20 chromatides-sœurs des dix univalents se séparent précocement pour former des structures en $X$ transitoires visibles à l'issue de la prophase I (Figure 4) avant leur ségrégation aléatoire. La cohésion des chomatides-sœurs semble donc un pré-requis à la formation des bivalents.
Figure 3. Les stades de la méiose chez Arabidopsis thaliana (méiocytes mâles, coloration DAPI). A- $\varepsilon$. Prophase I. A. Leptotène: les chromosomes sont diffus. B. Zygotène: les chromosomes commencent à se condenser. C. Pachytène: les chromosomes homologues sont appariés sur toute leur longueur. D. Diplotène: les chromosomes apparaissent plus diffus. $\varepsilon$. Diacinèse: les homologues ne sont plus associés qu'au niveau des chiasmas. F. Métaphase I: les cinq bivalents sont alignés sur la plaque métaphasique. G, H. Anaphase I: les chromosomes homologues se séparent. I. Métaphase II : les chromosomes s'alignent à nouveau en deux lots distincts sur les plaques métaphasiques. J. Anaphase II: les chromatides-sœurs se sont séparées; on distingue quatre lots chromosomiques. $\boldsymbol{K}$. Télophase II: les chromosomes se décondensent. $L$. Tétrade constituée de quatre microspores haploïdes.
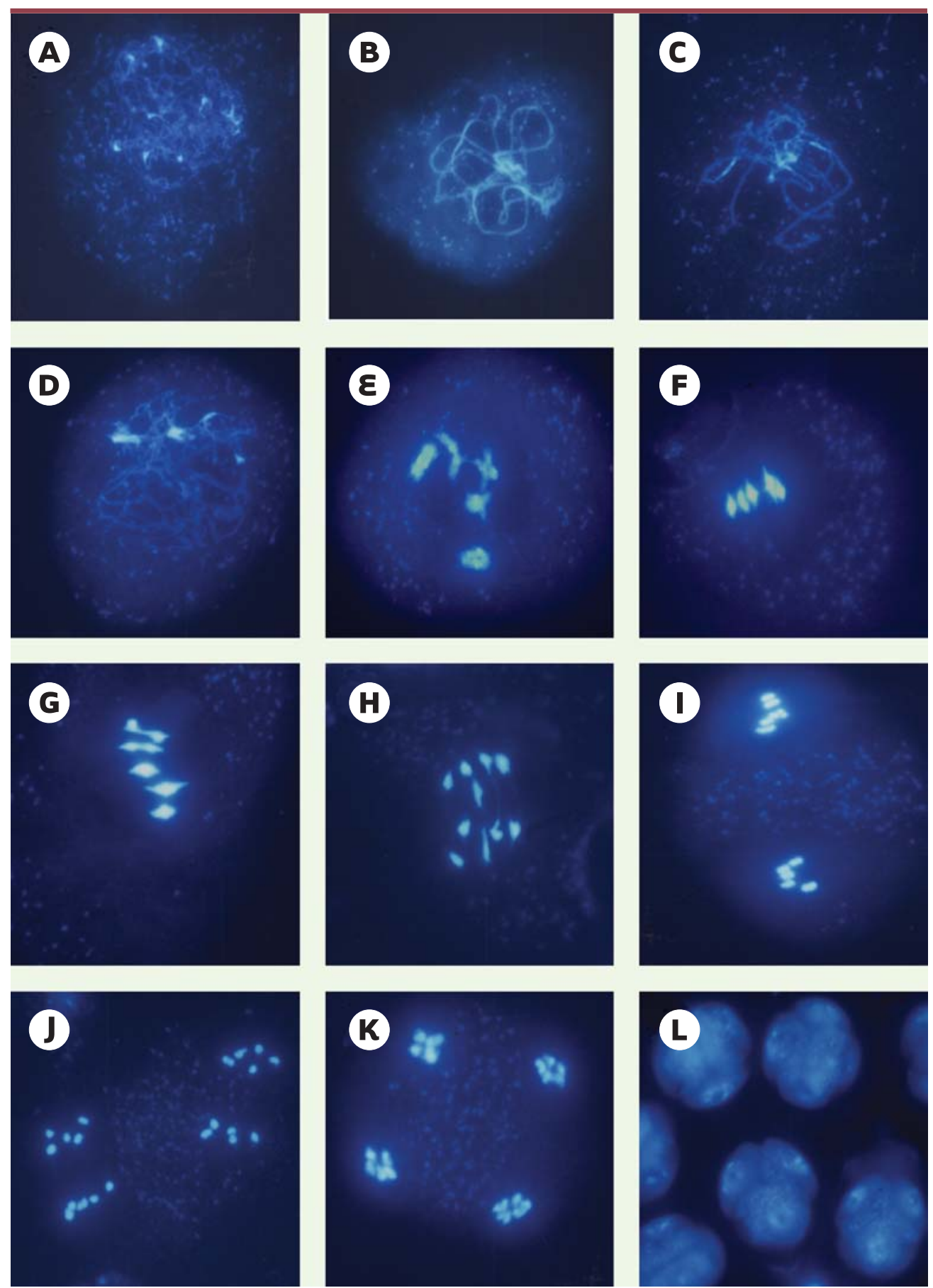
Comme la protéine Swil associée aux chromosomes durant les phases précoces de la méiose disparaît dès le stade leptotène, les auteurs [8] proposent que Swil intervienne dans l'établissement de la cohésion des chromatides-sœurs mais pas dans son maintien. La protéine Swil ne présente pas d'homologue connu chez d'autres espèces vivantes.

Le gène défectueux chez les mutants difl ou synl d'Arabidopsis code pour un homologue de la protéine Rad2l impliquée dans la cohésion des chromatides-sœurs chez Schizosaccharomyces pombe. Chez les plantes mutantes difl/synl, une majorité d'univalents sont discernables à l'issue de la prophase I. Les nombreuses entités chromosomiques ( $\geq 20$ ) observées en fin de télophase I suggèrent une séparation précoce des chromatides sœurs dès ce stade, éventuellement accompagnée de fragmentation chromosomique (voir plus loin) [9, 10]. Le phénotype des plantes difl/synl est restreint à la méiose, à la différence des mutants rad21 de $S$. pombe dont la croissance végétative est fortement affectée.

Le phénotype des mutants mâles swil-2 et des mutants difl/synl indique clairement que la cohésion des chromatides sœurs conditionne la formation des bivalents et que leur séparation précoce, avant la méiose II, est préjudiciable à la ségrégation correcte des chromosomes homologues.

\section{Les fragmentations chromosomiques}

Un homologue des gènes MECI (mitosis entry checkpoint) de levure ou ATM (ataxia telangiectasia-mutated kinase) de mammifères a été identifié chez Arabidopsis. La mutation de ce gène chez l'homme est responsable de l'ataxietélangiectasie, maladie qui entraîne, entre autres symptômes, un retard de croissance, une radiosensibilité, une stérilité et un risque élevé de cancers $(\rightarrow)$. ATM intervient dans les mécanismes de surveillance du cycle cellulaire (fonction de «point de contrôle ») qui peuvent être activés en réponse à certains dommages causés à l'ADN (cassures double-brin de l'ADN). La méiose ne s'interrompt pas chez la plupart des mutants méiotiques considérés jusqu'ici, ce qui suggère l'absence de point de contrôle du cycle cellulaire méiotique chez Arabidopsis. Néanmoins, les mutants atm d'Arabidopsis sont partiellement stériles, et présentent des figures méiotiques anormales: pontages chromosomiques entre bivalents en fin de prophase I, et présence de fragments chromosomiques lors des deux divisions méiotiques. La recombinaison méiotique atteint cependant la même fréquence chez le mutant atm que dans une plante sauvage. Les auteurs suggèrent qu'atm joue un rôle dans le contrôle du déroulement correct des événements de recombinaison, les événements aberrants (voir pontages) donnant lieu aux fragmentations décrites.

Deux autres mutants, mcdl et difl/synl, sont associés à des fragmentations chromosomiques, sans arrêt consécutif de la méiose. La fragmentation est visible dès le stade diplotène, à l'issue d'un stade pachytène normal chez le mutant modl [11]. Le gène correspondant à la mutation mcdl n'est pas identifié à ce jour. Le mutant difl/synl présente aussi un phénotype de fragmentation puisque les auteurs comptent parfois plus de 20 entités chromosomiques distinctes dès l'anaphase I.

Ces fragmentations chromosomiques pourraient refléter la rupture de bivalents soumis à des événements de $(\rightarrow) \mathrm{m} / \mathrm{s}$ 1999, n० 10 , p. 1086 recombinaison aberrants (pontages inter-homologues chez atm) ou l'absence de réparation des cassures double-brin induites par la protéine Spoll du fait de la dissociation précoce des chromatidessœurs (mutants syn 1). Les dix univalents régulièrement observés chez le mutant $d m c l$ semblent néanmoins intacts, soit qu'ils n'aient pas subi de cassure, soit que les cassures aient été réparées indépendamment de Dmcl.
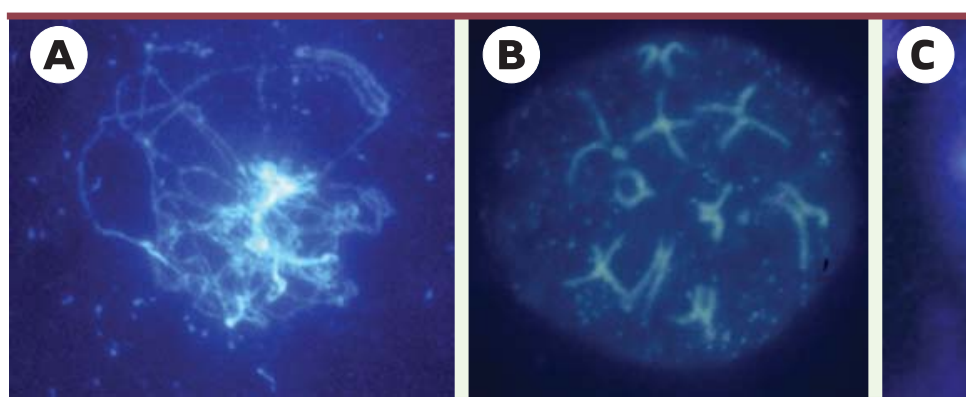

Figure 4. Trois mutants méiotiques d'Arabidopsis thaliana (méiocytes mâles, coloration DAPI). A. Prophase I chez le mutant spoll: il n'y a jamais d'appariement visible des chromosomes homologues chez le mutant spoll-1. $\boldsymbol{B}$. Le mutant swil-2 présente une séparation précoce des chromatides-sœurs, visible dès la fin de la prophase I. $C$. II n'y a pas formation de bivalents chez le mutant $d m c l, 10$ univalents ségrègent au hasard (le même type d'image est observé pour le mutant spoll).

\section{La séparation des chromosomes homologues}

La séparation des chromosomes homologues lors de l'anaphase I est défectueuse chez le mutant ask 1 [12]. La mutation résulte d'une insertion dans un gène qui code probablement pour un composant du système d'ubiquitinylation (homologue de Skpl chez la levure). Ce système 
cible spécifiquement des protéines définies en vue de leur protéolyse ultérieure. En conséquence, les auteurs proposent que Askl soit responsable de la dégradation de structures qui connectent les chromosomes homologues, comme le complexe synaptonémal ou les chiasmas.

\section{Méiose à 3 divisions}

Chez les mutants $m s 5 / t d m l$, à l'issue de la méiose, les tétrades contiennent généralement 8 microspores. Les deux divisions de méiose se déroulent normalement, mais sont immédiatement suivies d'une troisième division sans réplication préalable. Les chromatides s'étirent alors vers les deux pôles du fuseau achromatique avant de se répartir au hasard dans les nouveaux noyaux ainsi formés. Le gène dont la mutation est responsable de ce phénotype code pour une protéine de fonction inconnue [11, 13].

\section{Le cas des plantes polyploïdes}

Il est difficile de considérer la méiose chez les plantes sans évoquer le cas des plantes polyploïdes. Peu répandue chez les animaux, la polyploïdie concernerait 40 à $70 \%$ des plantes à fleurs et semblerait avoir joué un rôle majeur dans leur évolution et leur diversification [14]. Particulièrement représentées chez les espèces cultivées, les plantes polyploïdes sont souvent plus vigoureuses. Les allopolyploïdes, notamment, sont des hybrides qui résultent de la mise en présence de deux copies des génomes d'espèces différentes. Le doublement de chaque génome parental observé dans les hybrides stables permet à chaque chromosome de trouver un partenaire lors de la méiose; ainsi sont surmontés les problèmes de nombre et de synténie (conservation de l'organisation des gènes le long des chromosomes) des chromosomes, cause de stérilité chez les hybrides non doublés. Lors de la méiose, on observe généralement chez les allopolyploïdes stables la formation d'un nombre de bivalents équivalent à la somme des bivalents de chaque espèce diploïde parentale.

Ces observations supposent l'existence d'un mécanisme qui permet, lors de l'appariement des chromosomes, la reconnaissance des partenaires strictement homologues, évitant ainsi les interactions entre chromosomes apparentés (homéologues). À l'appui de cette hypothèse, la mutation $p h l$ isolée dans le blé (allohexaploïde $2 n=6 x$ = 42) autorise la recombinaison entre chromosomes homéologues à des fréquences similaires à celles observées pour des chromosomes homologues et s'accompagne de la formation récurrente de polyvalents lors de la méiose $[15,17]$. De façon surprenante, malgré un contexte chromosomique complexe, la méiose est plus rapide chez les plantes polyploïdes. En fait, chez ces plantes, les centromères et les chromosomes homologues sont associés avant la méiose, pendant le développement floral, ce qui n'est pas le cas chez les plantes diploïdes. En l'absence de Phl, s'il n'y a plus appariement des chromosomes homologues à l'état somatique, les centromères demeurent associés dans les plantes polyploïdes. Phl semble donc stimuler les interactions homologues déclenchées au niveau des centromères dans un contexte polyploïde, limitant ainsi les interactions homéologues et facilitant l'établissement de bivalents lors d'une méiose ultérieure [18]. Le nombre de gènes candidats restant à évaluer dans la région délétée chez le mutant phl est de plus en plus réduit, ce qui laisse espérer son identification dans un avenir proche [19].

\section{Conclusions}

La méiose chez les plantes semble se dérouler selon les mêmes modalités que dans la plupart des organismes diploïdes: I'établissement de bivalents stables est un pré requis à la ségrégation correcte des chromosomes homologues et dépend de la cohésion des chromatides sœurs, de la présence du complexe synaptonémal et de la mise en route de la recombinaison homologue. L'identification de nombreux mutants méiotiques végétaux non encore caractérisés permettra une définition plus fine des spécificités des plantes dans ce processus de la méiose. Il est néanmoins important de souligner que les défauts constatés chez les mutants décrits ci-dessus n'entraînent généralement pas leur stérilité complète. L'avancement imperturbable dans la méiose alors que des étapes-clés sont court-circuitées, l'existence de structures chromosomiques aberrantes, l'adaptation à la présence simultanée de génomes d'espèces différentes supposent que les cellules végétales présentent une tolérance élevée aux altérations de leur génome. Cette plasticité ouvre des champs d'étude que nous sommes loin de maîtriser à ce jour. $\diamond$

\section{SUMMARY}

Molecular mechanisms of meiosis in plants

Meiosis is a key step in diploid sexual reproduction. Apart from its cytological description, the molecular mechanisms involved in this specialized cell division are being deciphered in plants thanks to the model plant Arabidopsis thaliana. While some meiotic mutants of Arabidopsis confirm the central role of functions that have been described either in yeast or in mice, others led to the identification of previously unknown genes. Numerous plants also exist as polyploids, which represent a special case with regard to meiosis. $\diamond$ 


\section{RÉFÉRENCES}

1. The Arabidopsis Genome Initiative. Analysis of the genome sequence of the flowering plant Arabidopsis thaliana. Nature 2000; 408 : 796-815.

2. Ross KJ, Fransz P, Jones GH. A light microscopic atlas of meiosis in Arabidopsis thaliana. Chrom Res 1996; 4: 507-16.

3. Caryl AP, Armstrong SJ, Jones GH, Franklin FC. A homologue of the yeast $\mathrm{HOPl}$ gene is inactivated in the Arabidopsis meiotic mutant asyl. Chromosoma 2000; 109: 62-71.

4. Copenhaver GP, Browne WE, Preuss D. Assaying genomewide recombination and centromere functions with Arabidopsis tetrads. Proc Natl Acad Sci USA 1998; 6: 247-52.

5. Masson JE, Paszkowski J. Arabidopsis thaliana mutants altered in homologous recombination. Proc Natl Acad Sci USA 1997; 14: 11731-5.

6. Grelon M, Vezon D, Gendrot
G, Pelletier G. AtSP011-1 is necessary for efficient meiotic recombination in plants. EMBO J 2001; 20 : 589-600

7. Couteau F, Belzile F, Horlow C, Grandjean O, Vezon D, Doutriaux MP. Random chromosome segregation without meiotic arrest in both male and female meiocytes of a dmcl mutant of Arabidopsis. Plant Cell 1999; 11: 1623-34.

8. Mercier R, Vezon D, Bullier $\varepsilon$, et al. SWITCHI (SWI): a novel protein required for the establishment of sister chromatid cohesion and for bivalent formation at meiosis. Genes Dev 2001; 15: 1859-71.

9. Bhatt AM, Lister C, Page T, et al. The DIFl gene of Arabidopsis is required for meiotic chromosome segregation and belongs to the REC8/RAD21 cohesin gene family. Plant J 1999; 19: 463-72.

10. Bai X, Peirson BN, Dong F, Xue C, Makaroff CA. Isolation and characterization of SYN1, a RAD21-like gene essential for meiosis in Arabidopsis. Plant Cell 1999; 11: 417-30.

11. Ross KJ, Fransz P, Armstrong SJ, et al. Cytological characterization of four meiotic mutants of Arabidopsis isolated from TDNA-transformed lines. Chromosome Res 1997; 5 : 551-9.

12. Yang $M, H u Y$, Lodhi $M$, McCombie WR, Ma H. The Arabidopsis SKPl-likel gene is essential for male meiosis and may control homologue separation. Proc Natl Acad Sci USA 1999; 96: 11416-21.

13. Glover J, Grelon M, Craig $S$, Chaudhury A, Dennis $\varepsilon$. Cloning and characterization of MS5 from Arabidopsis: a gene critical in male meiosis. Plant J 1998; 15 : 345-56.

14. Otto SP, Whitton J. Polyploid incidence and evolution. Annu Rev Genet 2000; 34: 401-37.

15. Sears $\varepsilon R$. Genetic control of chromosome pairing in wheat. Annu Rev Genet 1976; 10: 31-51.

16. Dubcovsky J, Luo M,
Dvorak J. Differentiation between homoeologous chromosomes $1 \mathrm{~A}$ of wheat and $1 A m$ of Triticum monococcum and its recognition by the wheat Phl locus. Proc Natl Acad Sci USA 1995; 92: 6645-9.

17. Vega JM, Feldman M. Effect of the pairing gene $P h l$ and premeiotic colchicine treatment on intra- and interchromosome pairing of isochromosomes in common wheat. Genetics 1998; 150 : 1199-208.

18. Martinez-Perez $\varepsilon$, Shaw $P$, Moore G. The Phl locus is needed to ensure specific somatic and meiotic centromere association. Nature 2001; 411: 204-7.

19. Foote T, Roberts M, Kurata N, Sasaki T, Moore G. Detailed comparative mapping of cereal chromosome regions corresponding to the Phl locus in wheat. Genetics 1997; 147: 801-7.

\section{TIRÉS À PART}

M.P. Doutriaux

\section{Bon de commande}

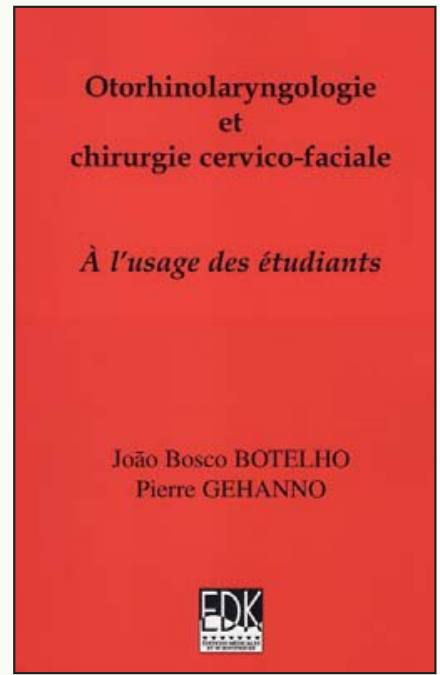

ISBN: 2-84254-075-1 160 pages
À retourner à EDK, 10 Villa d'Orléans - 75014 PARIS

Tél.: 0153910606 - Fax: 0153910607 - E-mail : editorial@edk.fr

NOM:

Prénom:

Adresse:

Code postal:

Ville

Pays:

Fonction:

Je souhaite recevoir l'ouvrage ORL et chirurgie cervico-faciale: $30 €+3 €$ de port $=33 €$ TTC

en exemplaire, soit un total

$€$

$\square$ Par chèque, à l'ordre de $\mathbf{E} \mathbf{D} \mathbf{K}$

Par carte bancaire: $\quad \square$ Visa $\square$ Eurocard/Mastercard $\square$ American Express

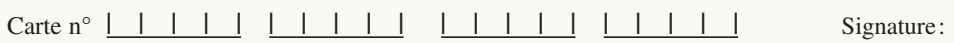

Date d'expiration: 